\title{
Left heart function evaluation of patients with essential hypertension and paroxysmal atrial fibrillation by two-dimensional speckle tracking imaging combined with real-time three-dimensional ultrasound imaging
}

\author{
Fengxia Jiang ${ }^{1 \#}$, Yiwen Chen ${ }^{2 \#}$, Liu Wu ${ }^{3}$, Yi Zhang ${ }^{1}$, Jianxin Liu ${ }^{1}$, Xiaofeng Sun ${ }^{1}$, Jueying Li ${ }^{1}$, \\ Mingfeng Mao', Shunshi Yang ${ }^{1}$
}

${ }^{1}$ Department of Ultrasound, The Central Hospital of Wuhan, Tongji Medical College, Huazhong University of Science and Technology, Wuhan, China; ${ }^{2}$ Division of Cardiovascular Medicine, Department of Internal Medicine, Wuhan Hospital of Traditional Chinese Medicine, Wuhan, China; ${ }^{3}$ Department of Cardiovascular Medicine, The Central Hospital of Wuhan, Tongji Medical College, Huazhong University of Science and Technology, Wuhan, China

Contributions: (I) Conception and design: S Yang, F Jiang, Y Chen; (II) Administrative support: S Yang, F Jiang, Y Chen; (III) Provision of study materials or patients: F Jiang, Y Chen, L Wu, Y Zhang, J Liu; (IV) Collection and assembly of data: F Jiang, Y Chen, X Sun, J Li; (V) Data analysis and interpretation: F Jiang, Y Chen, M Mao; (VI) Manuscript writing: All authors; (VII) Final approval of manuscript: All authors.

\#These authors contributed equally to this work.

Correspondence to: Shunshi Yang, MM. Department of Ultrasound, The Central Hospital of Wuhan, Tongji Medical College, Huazhong University of Science and Technology, NO.26 Shengli Street, Jiangan District, Wuhan 430014, China. Email: yangzai19610816@163.com.

Background: This study aims to assess the value of two-dimensional speckle tracking imaging (2D-STI) combined with real-time 3-dimensional echocardiography (RT-3DE) in evaluating left heart function in patients with combined ETH and PAF.

Methods: A prospective study was designed, and the research period was from January 2017 to January 2020. A PAF observation group comprised of patients with ETH and PAF, a PAF control group of patients with ETH but without PAF, and a healthy volunteer group referred to as the healthy group, each comprised of 50 patients was established. All patients underwent routine ultrasound imaging examination using 2D-STI and RT-3DE. The interventricular septum thickness (IVST), left and right atrioventricular diameter (LAD), left ventricular posterior wall thickness (PWT), left ventricular ejection fraction (LVEF), the mean left atrium peak strain ( $\mathrm{mSs}, \mathrm{mSe}, \mathrm{mSa}$ ), strain rate (mSRs, mSRe, mSRa), the left ventricular mass (LVM), left ventricular mass index (LVMI), and left atrial total emptying volume (LATEV), the left atrial volume index (LAVI), left atrial total emptying fraction (LATEF), left atrium expansion index (LAEI), left atrium passive ejection fraction (LAPEF), and left atrial active ejection fraction (LAAEF) were calculated. Patients in the PAF observation group received telmisartan combined with rosuvastatin after diagnosis and 12- and 24-month-follow up visits were conducted. During these visits, 2D-STI combined with RT-3DE was used to evaluate cardiac function and each patient's blood pressure was monitored.

Results: Patients in the PAF observation group had a significantly longer course of ETH than patients in the PAF control group $(13.5 \pm 4.4 v s$. $10.32 \pm 5.6, \mathrm{P}=0.002)$. The comparison of routine 2D-STI and RT$3 \mathrm{DE}$ ultrasound indexes among the three groups also showed significant differences $(\mathrm{P}<0.01)$. During the 2-year follow-up period, the IVST, LAD, PWT, and LVEF of remaining patients significantly improved as treatment progressed $(\mathrm{P}<0.05)$. The LAVI, LATEF, and LAEI also improved significantly as the treatment progressed $(\mathrm{P}<0.05)$. After 12 months of treatment, the recurrence rate of atrial fibrillation was $16.33 \%(8 / 45)$ and after 24 months this was $34.21 \%(13 / 38)$.

Conclusions: Cardiac function could be evaluated comprehensively by 2D-STI combined with RT-3DE in patients with ETH combined with PAF at initial diagnosis and follow-up. 
Keywords: Essential hypertension (ETH); paroxysmal atrial fibrillation (PAF); heart function; two-dimensional speckle tracking imaging (2D-STI); real-time three dimensional

Submitted Nov 16, 2020. Accepted for publication Jan 18, 2021.

doi: $10.21037 /$ jtd-20-3577

View this article at: http://dx.doi.org/10.21037/jtd-20-3577

\section{Introduction}

Essential hypertension (ETH) is a commonly encountered clinical condition worldwide (1). A 2016 study (2) published in 'The Lancet' showed that there were 594 million ETH patients worldwide in 1975 , and this had increased to 1.1 billion in 2015. ETH is the most important risk factor for cardiovascular and cerebrovascular diseases (3) and may also be associated with functional damage to organs such as the heart and brain $(3,4)$. Paroxysmal atrial fibrillation (PAF) is a common clinical disorder. Many small reentrant rings caused by the atrium-dominant reentrant ring cause the cardiac rhythm disorders of PAF patients (5). There is a high incidence of ETH in patients with PAF and when combined, there is an increased risk of arterial embolism, heart failure, secondary stroke complications, and death (5-7).

At present, there are few studies focusing on cardiac function in patients with ETH combined with PAF. Ultrasonography is the most used method for evaluating cardiac function and two-dimensional speckle tracking imaging (2D-STI) has been widely used in the evaluation of cardiovascular disease. As a relatively new method to evaluate cardiac function, 2D-STI not only eliminates the dependence of tissue Doppler on the angle of the sound beam but is also not interfered with by the traction of the surrounding myocardium and heart movement $(8,9)$. However, the spatial conformation of the atria and ventricles are not a regular geometric shape (10) and the results of two-dimensional Doppler ultrasound measurement are prone to errors (11). Real-time three-dimensional (RT$3 \mathrm{DE}$ ) ultrasound does not need to assume the geometry of the heart (12) and can display the heart space structure, movement law. And adjacent relationship as well as measuring the volume of the heart in real time $(13,14)$. Compared with the left atrial volume measured by MRI and CT, RT-3DE measurement has better repeatability and inter observer repeatability. Therefore, combined 2D-STI and RT-3DE can more comprehensively evaluate changes to cardiac structure and function.
In this study, 2D-STI combined with RT-3DE was used to evaluate cardiac function in patients with ETH combined with PAF, and the changes of cardiac function in the 24 months following treatment were observed. The aim was to provide a new method for disease assessment and curative effect evaluation in patients with ETH combined with PAF. We present the following article in accordance with the STROBE reporting checklist (available at http:// dx.doi.org/10.21037/jtd-20-3577).

\section{Methods}

\section{Patients}

This prospective study was conducted from January 2017 to January 2020. A total of 150 cases were enrolled including 50 with ETH combined with PAF in the PAF observation group, 50 with ETH but not with PAF in the PAF control group, and 50 healthy volunteers in the healthy group. The inclusion criteria were as follows: (I) all subjects were followed up for 24 months after joining the study; (II) patients in the PAF observation group and PAF control group all met the clinical diagnosis of ETH $(15,16)$; (III) patients in the PAF observation group were in compliance with the clinical diagnosis; (IV) after physical examination, the patients in Healthy group were completely healthy; (V) the age range was 50-65 years old. The exclusion criteria were: (I) patients with respiratory and digestive function, blood circulation and serious hematological diseases, and patients with hyperthyroidism, malignant tumors, disorders of glucose metabolism or coronary atherosclerosis, and patients with valvular diseases or other heart diseases; (II) patients who were unable to complete the study.

\section{Ethical approval}

All procedures performed in this study involving human participants were in accordance with the Declaration of Helsinki (as revised in 2013). The study was approved by the Clinical Ethics Committee of The Central Hospital of 
Wuhan (No. CHW201609). All participating patients had been informed of its content and signed informed consent.

\section{Image examination}

\section{Instrument}

A Philips iE Elite color Doppler ultrasound diagnostic equipment, equipped with $2 \mathrm{D}$ phased array probe s5-1 (frequency 2.0-3.5 MHz), real-time three-dimensional probe $\mathrm{x}$ 5-1 (frequency 1.0-3.0 MHz) was used.

\section{Image acquisition}

Patients were placed in the left decubitus position and instructed to breathe calmly. The ECG of the chest lead was synchronized and two-dimensional dynamic images of the left ventricular long axis, the apical four chamber heart, and the apical two chamber cardiac section collected. The left ventricular anteroposterior diameter (LAD) was measured in the left ventricle by long axis measurement and a clear apical four chamber image was obtained after the 3-dimensional X5-1 probe commenced. After adjusting the gain, contrast, brightness, and other factors to produce an appropriate left atrium image, the patient was asked to hold their breath at the end of a breath and "Full-volume" imaging was performed. The best full-volume threedimensional images were collected for four consecutive cardiac cycles.

\section{Image analysis}

Using a QLAB workstation, the sampling points were placed in the atrium septum of the mitral annulus, left atrial side wall, and the left roof of the mitral annulus in the apical four chamber and apical two chamber heart images, respectively. The left atrial strain and strain rate curves were automatically analyzed in the aCMQ mode (Figure 1A) and the left atrial systolic peak strain (Ss, Se, Sa) and peak strain rate (SRs, SRe, SRa) were measured in the left ventricular systolic phase, the early diastolic stage of left ventricle, and the late left ventricular diastolic function. The 2D mean peak strain $(\mathrm{mSs}, \mathrm{mSe}, \mathrm{mSa})$ and strain rate (mSRs, $\mathrm{mSRe}$, and $\mathrm{mSRa}$ ) were calculated, and all parameters were measured 5 times (17).

Using the 3DQAdvance mode of the QLAB workstation, the left ventricular mass (LVM), left ventricular mass index (LVMI), left ventricular ejection fraction (LVEF), and left atrial total emptying volume (LATEV) were analyzed at the end of the systolic phase on the atrial septum and lateral wall at the apical four chamber and two chamber section mitral annulus. A reference point was drawn from the roof of the apical two-chamber view and left atrial time-volume curve was automatically drawn (Figure 1B). The left atrial volume at each time was measured and normalized to the left atrial volume index through the body surface area and the left atrial maximum volume index $\left(\mathrm{LAVI}_{\max }\right)$ and end diastolic left atrial minimum volume index $\left(\mathrm{LAVI}_{\text {min }}\right)$ were immediately measured before mitral valve opening. The left atrial systolic volume index $\left(\mathrm{LAVI}_{\mathrm{p}}\right)$ was measured by $\mathrm{P}$ wave front and the left atrial total ejection fraction (LATEF) and left atrial active ejection fraction (LAAEF) were calculated (18):

$$
\begin{aligned}
& \text { LATEF }=\left(\mathrm{LAV}_{\text {max }}-\mathrm{LAV}_{\text {min }}\right) / \mathrm{LAV}_{\text {max }} \times 100 \% \\
& \mathrm{LAEI}=\left(\mathrm{LAV}_{\text {max }}-\mathrm{LAV}_{\text {min }}\right) / \mathrm{LAV}_{\text {min }} \times 100 \% \\
& \mathrm{LAPEF}=\left(\mathrm{LAV}_{\text {max }}-\mathrm{LAV}_{\mathrm{p}}\right) / \mathrm{LAV}_{\text {max }} \times 100 \% \\
& \mathrm{LAAEF}=\left(\mathrm{LAV}_{\mathrm{p}}-\mathrm{LAV}_{\text {min }}\right) / \mathrm{LAV}_{\mathrm{p}} \times 100 \%
\end{aligned}
$$

\section{Medical treatment}

Patients in the PAF observation group received routine antiarrhythmic treatment including amlodipine, felodipine and nifedipine. This combination therapy has been reported in recent years (19-21). On this basis, rosuvastatin (Rosuvastatin Calcium Tablets, Zhejiang Hai Zheng pharmaceutical Limited by Share Ltd., Chinese medicine: H20143337) treatment, $5 \mathrm{mg}$, QD, po and telmisartan (Mikacin, Shanghai, Imported Drug Registration Certificate No. H20090416, specifications: $80 \mathrm{mg} /$ tablets) treatment, oral, 1 times/d, 30-80 $\mathrm{mg}$ were initiated. If a patient chose to continue in the study their condition was observed after each 12 months of continuous treatment.

\section{Follow-up and data collection}

An electronic summary table to register the patient's treatment information and observation index data was established. When the patient was hospitalized, we conducted a full communication study emphasizing the necessity of follow-up for 24 months after the image examination. The medical records of all patients were recorded in detail.

\section{Outcome measures}

\section{Primary outcome}

These were the index measurements in the PAF observation group (patients in this group were treated with telmisartan combined with rosuvastatin commenced); blood pressure, 


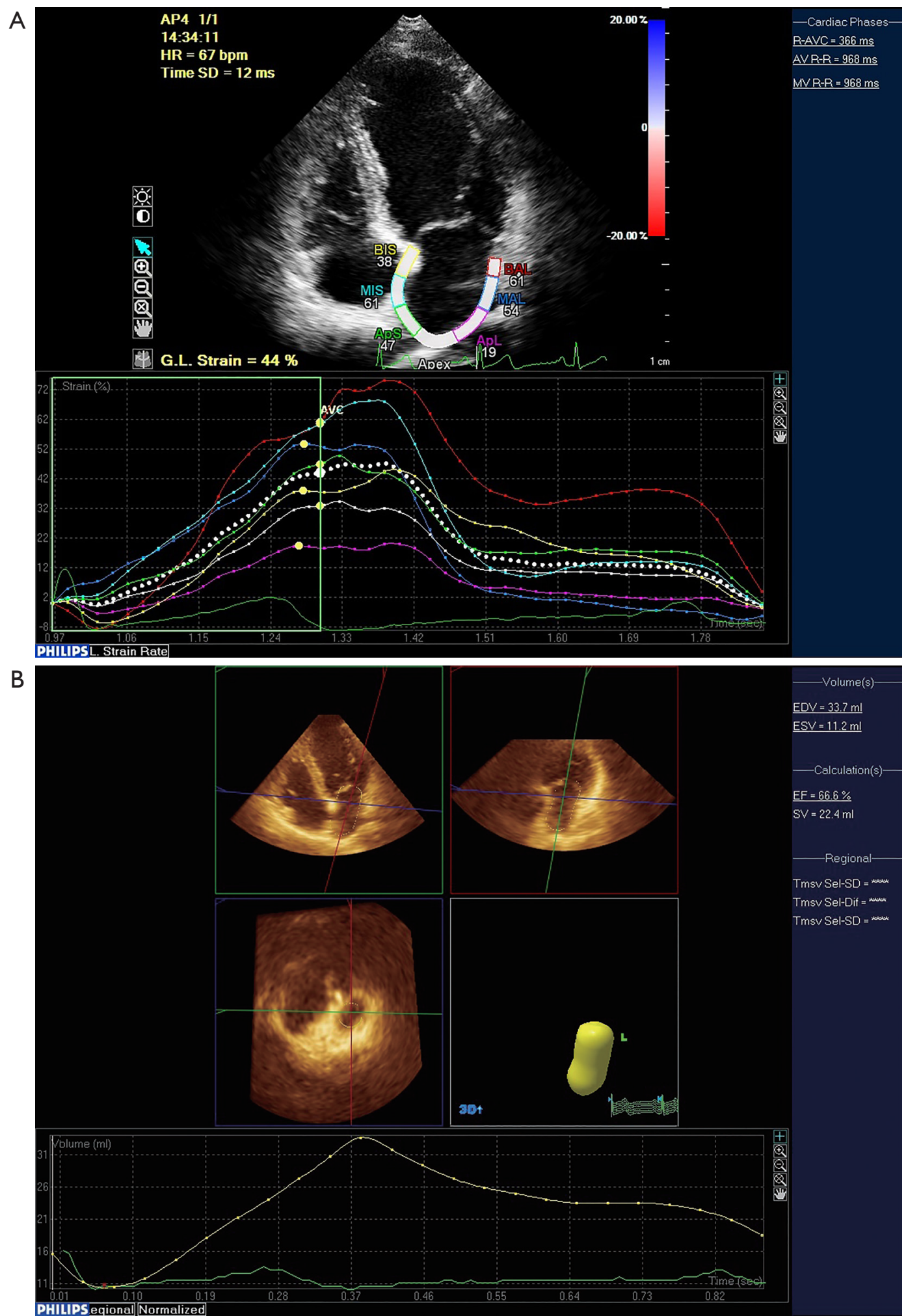

Figure 1 Examples of image analysis in the QLAB workstation. (A) The left atrial strain and strain rate curves were automatically analyzed in the aCMQ mode. (B) After entering the 3DQAdvance mode, a reference point was drawn from the roof of the apical two-chamber view, and left atrial time-volume curve was automatically drawn. 


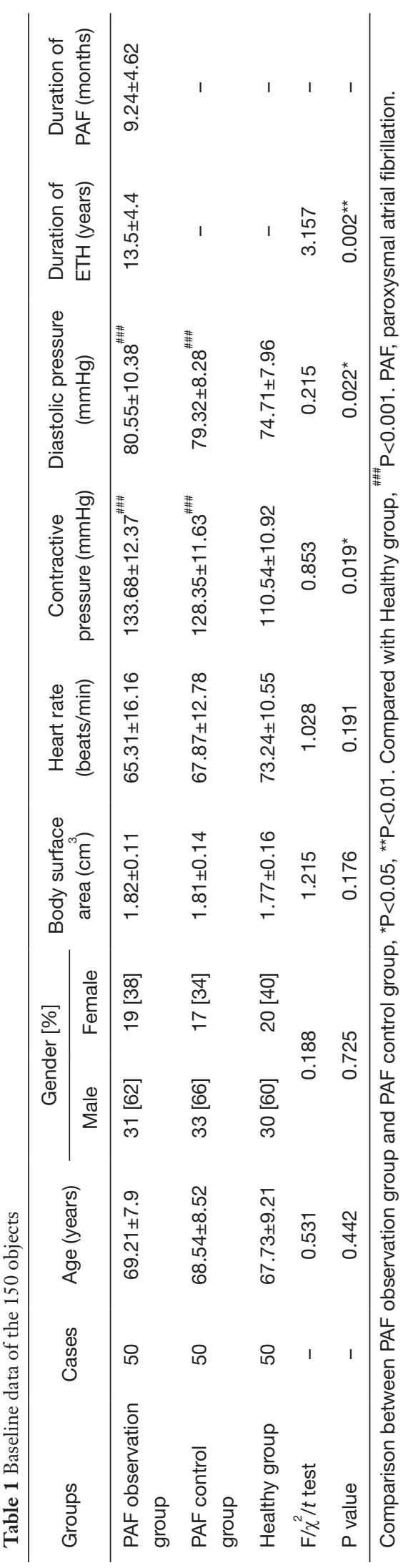

IVST, LAD, PWT, LVEF, LATEF, LAEI, mSs, mSe, mSRs, and mSRe. The index measurements were recorded at base, 12 months, and 24 months after treatment.

\section{Secondary outcomes}

These were the differences in the following ultrasound index measurements in each group; IVST, LAD, PWT, LVM, LVMI, LVEF, LATEV, LAVI, LAAEF, LATEF, mSs, $\mathrm{mSe}, \mathrm{mSa}$, mSRs, mSRe, and mSRa.

\section{Statistical analysis}

SPSS 24.0 (SPSS Inc. Chicago, IL, USA) was used for statistical analysis. The count data was represented by examples (percent) (n (\%)); the theoretical number $\mathrm{T} \geq 5$ and the total sample size $\mathrm{n} \geq 40$ using Pearson $\chi^{2}$ test; the theoretical number $\mathrm{T}<5$ but $\mathrm{T} \geq 1$ and $\mathrm{n} \geq 40$ using the continuous correction of $\chi^{2}$ test; and the theoretical number $\mathrm{T}<1$ or $\mathrm{n}<40$, using Fisher's exact test. The measurement data obeying normal distribution were expressed as mean \pm standard deviation $(\bar{x} \pm s)$ indicating the comparison between groups was performed with an independent sample t test, and the comparison within a group was performed with a paired sample $t$ test. Repeated measurement analysis of variance was used to compare multiple sets of measurement data and data that did not obey the normal distribution were expressed as $\mathrm{P}_{50}\left(\mathrm{P}_{25}, \mathrm{P}_{75}\right)$ and the Mann-Whitney $\mathrm{U}$ test used for comparison. The Mann-Whitney $\mathrm{U}$ test was also used for the difference in distribution of grade data and both levels of $\alpha=0.05$ were used for the test level. Statistical significance was set at $\mathrm{P}<0.05$.

\section{Results}

\section{Baseline}

Compared with patients in the PAF control group, those in the PAF observation group had a significantly longer course of ETH $(13.5 \pm 4.4 v s .10 .32 \pm 5.6, \mathrm{P}=0.002)$ and the blood pressure of patients in PAF control and PAF observation groups was significantly higher than that in the healthy group $(\mathrm{P}<0.001)$. The basic clinical data of all 150 patients is shown in Table 1.

\section{Comparison of ultrasound indexes of the three groups}

As shown in Table 2, the comparison of IVST, LAD, PWT, and LVEF among the three groups showed significant 
Table 2 Data of routine ultrasound examination in the 150 objects

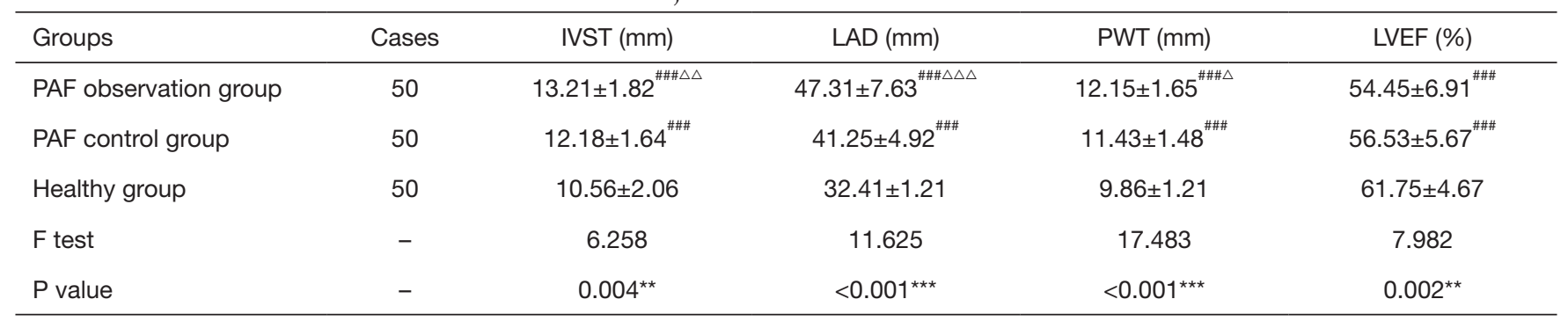

Comparison among PAF observation group, PAF control group, and healthy group, ${ }^{\star *} \mathrm{P}<0.01,{ }^{* \star *} \mathrm{P}<0.001$. Compared with healthy group,

${ }_{\# \# \#} \mathrm{P}<0.001$. Compared with PAF control group, ${ }^{\Delta} \mathrm{P}<0.05,{ }^{\triangle \Delta} \mathrm{P}<0.01,{ }^{\triangle} \Delta{ }^{\mathrm{P}}<0.001$. IVST, interventricular septal thickness; LAD, left atrial diameter; PWT, posterior wall thickness; LVEF, left ventricular ejection fraction; PAF, paroxysmal atrial fibrillation.

Table 3 Data of 2D-STI examination in the 150 patients

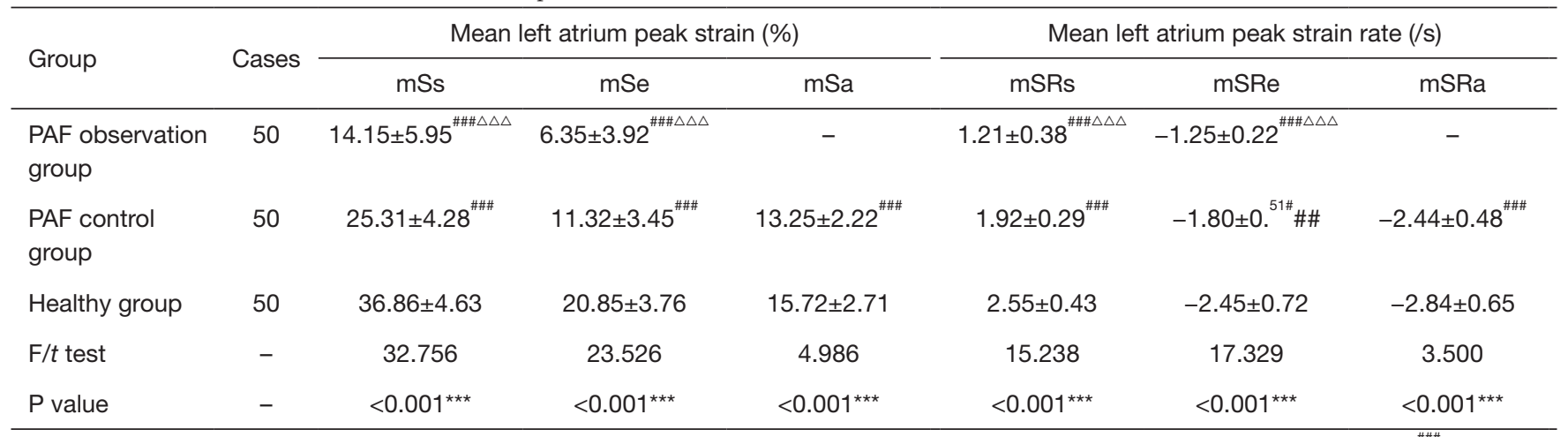

Comparison among PAF observation group, PAF control group, and healthy group, ${ }^{\star * \star} \mathrm{P}<0.001$. Compared with healthy group, ${ }^{\# \#} \mathrm{P}<0.001$.

Compared with PAF control group, ${ }^{\triangle \triangle \Delta} \mathrm{P}<0.001$. 2D-STI, two-dimensional speckle tracking imaging; PAF, paroxysmal atrial fibrillation.

differences $(\mathrm{P}<0.01)$. Compared with the healthy group, patients in the PAF control and PAF observation groups had significantly higher IVST, LAD, PWT $(\mathrm{P}<0.001)$, and significantly lower LVEF $(\mathrm{P}<0.001)$. PAF control group patients had significantly higher IVST, LAD and lower PWT than the PAF observation group $(\mathrm{P}<0.05)$.

As shown in Table 3, comparison of the mean left atrium peak strain and mean left atrium peak strain rates among the three groups also showed significant differences $(\mathrm{P}<0.01)$. Compared with the healthy group, the PAF control and PAF observation groups had significantly lower $\mathrm{mSs}, \mathrm{mSe}$, $\mathrm{mSa}$, and $\mathrm{mSRs}(\mathrm{P}<0.001)$, and significantly higher $\mathrm{mSRe}$ and mSRa $(\mathrm{P}<0.001)$. The mSe and mSRs of patients in the PAF observation group were significantly lower than the PAF control group $(\mathrm{P}<0.001)$, while the $\mathrm{mSR}$ a was significantly higher $(\mathrm{P}<0.001)$.

As shown in Table 4, compared with the healthy group, the PAF observation and PAF control groups had a significantly higher LAVI and significantly lower LATEF and LAEI $(\mathrm{P}<0.001)$.

\section{Changes of ultrasound indexes of patients in the PAF observation group before and after treatment}

During the 2-year follow-up, a total of 12 patients left the study including five in the first and seven in the second years.

When compared with measurements obtained before treatment commenced, those obtained both at the 12- and 24-month follow ups showed significant and progressive improvement.

Table 5 shows progressive improvement in the IVST, LAD, PWT, and LVEF and Table 6 in the mean left atrium peak strain and mean peak left atrium strain rate. Table 7 shows the LAVI was significantly lower $(\mathrm{P}<0.05)$ and LATEF and LAEI were significantly higher $(\mathrm{P}<0.05)$ across the 24 months. 


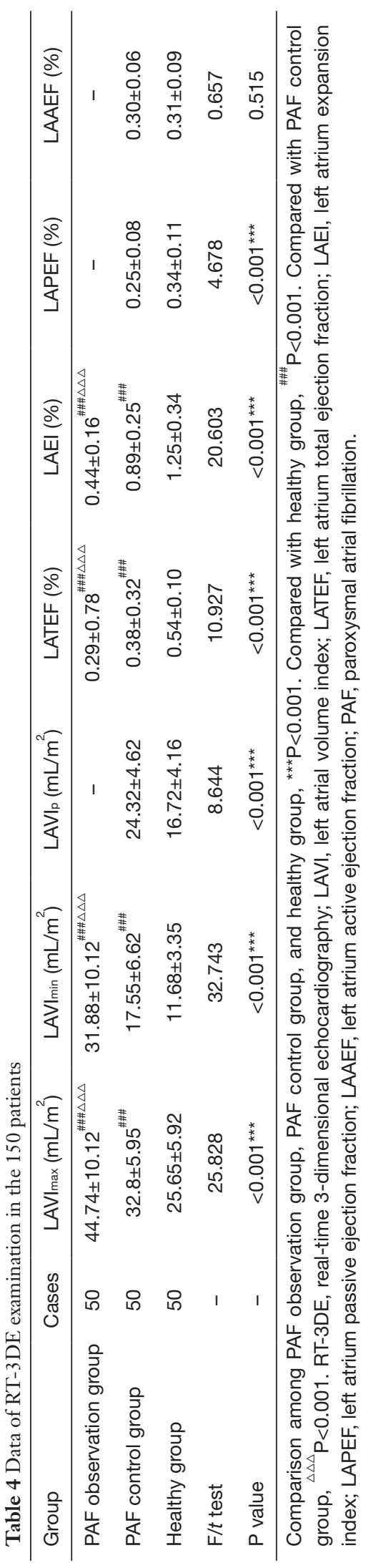

Blood pressure and atrial fibrillation rate of patients in the PAF observation group during follow-up

As shown in Table 8, the contractive pressure and diastolic pressure of patients after 24 months of treatment were significantly reduced compared with those before and after 12 months of treatment $(\mathrm{P}<0.01)$. After 12 months of treatment, the recurrence rate of atrial fibrillation was $16.33 \%(8 / 45)$ and after 24 months this was $34.21 \%(13 / 38)$.

\section{Discussion}

Cardiac insufficiency during diastole or systole in patients with ETH causes both ventricular and atrial pressures to change (22) resulting in the level of angiotensinconverting enzyme in atrial tissue to significantly increase and the density of angiotensin receptors to increase (23). Mediated by angiotensin receptor type I, calcium overload in myocardial cells increases, which in turn triggers atrial fibrillation. At the same time, angiotensin receptors can also influence angiotensin type I receptors causing atrial muscle hypertrophy. The accumulation of extracellular matrix proteins leads to obvious fibrosis of the atria, which affects conduction function and triggers atrial remodeling $(22,23)$.

In this study, atrial remodeling and decreased cardiac function in ETH combined with PAF patients were confirmed using 2D-STI and RT-3DE. By measuring the strain and strain rate of local atrial muscle, 2D-STI quantitative analysis could reflect left atrium remodeling (24) and RT-3DE could display the spatial structure of the heart in real time and three-dimensionally, the law of motion and its neighboring relationship, and heart volume in real time (25). Atas et al. (26) showed that the combined application of 2D-STI and RT-3DE could improve the accuracy of left atrial function evaluation. The three functions of the left atrium and related indicators $(27,28)$ are listed below:

(I) Function of 'storage': storing the blood returning from the pulmonary vein (left ventricular systole). LAVmax, LAVmin, LATEF, LAEI, mSs, and mSRs were used to evaluate this function.

(II) Function of 'transport': transporting blood in the pulmonary vein to the left ventricle (early left ventricular diastole). LAPEF, $\mathrm{mSe}$, and $\mathrm{mSRe}$ were used to evaluate this function.

(III) Function of 'Auxiliary Pump': contracting the left atrial myocardium actively to increase filling of the left ventricle by $20-30 \%$ (late diastole of the left 
Table 5 Data change of routine ultrasound examination of the patients in PAF observation group

\begin{tabular}{lccccc}
\hline Time & Cases & IVST $(\mathrm{mm})$ & LAD $(\mathrm{mm})$ & PWT (mm) & LVEF $(\%)$ \\
\hline Before treatment & 50 & $13.21 \pm 1.82$ & $47.31 \pm 7.63$ & $12.15 \pm 1.65$ & $54.45 \pm 6.91$ \\
Treated for 12 months & 45 & $12.88 \pm 1.55^{\# \# \#}$ & $43.35 \pm 5.87^{\# \#}$ & $11.49 \pm 1.52^{\#}$ & $57.25 \pm 5.58^{\#}$ \\
Treated for 24 months & 38 & $11.45 \pm 1.47^{\# \# \Delta \Delta \Delta}$ & $39.85 \pm 4.33^{\# \# \# \Delta \Delta}$ & $10.36 \pm 1.31^{\# \# \# \Delta \Delta}$ & $60.23 \pm 4.86^{\# \# \# \Delta \Delta}$ \\
F test & - & 5.897 & 7.112 & 5.314 & 7.035 \\
P value & - & $0.009^{\star \star}$ & $0.005^{\star \star}$ & $0.021^{\star}$ & $0.006^{\star \star}$ \\
\hline
\end{tabular}

Comparison among before treatment, treated for 12 months and treated for 24 months, ${ }^{\star} P<0.05$, ${ }^{\star \star} P<0.01,{ }^{* \star \star} P<0.001$. Compared with before treatment, ${ }^{\sharp} \mathrm{P}<0.05,{ }^{\# \#} \mathrm{P}<0.01,{ }^{\# \# \#} \mathrm{P}<0.001$. Compared with treated for 12 months, ${ }^{\Delta \Delta} \mathrm{P}<0.01,{ }^{\Delta \Delta} \mathrm{P}<0.001$. PAF, paroxysmal atrial fibrillation; IVST, interventricular septal thickness; LAD, left atrial diameter; PWT, posterior wall thickness; LVEF, left ventricular ejection fraction.

Table 6 Data change of 2D-STI examination of the patients in PAF observation group

\begin{tabular}{|c|c|c|c|c|c|}
\hline Time & Cases & \multicolumn{2}{|c|}{ Mean left atrium peak strain (\%) } & \multicolumn{2}{|c|}{ Mean peak left atrium strain rate (/s) } \\
\hline Before treatment & 50 & $14.15 \pm 5.95$ & $6.35 \pm 3.92$ & $1.21 \pm 0.38$ & $-1.25 \pm 0.22$ \\
\hline Treated for 12 months & 45 & $20.75 \pm 5.33^{\# \# \#}$ & $9.25 \pm 3.62^{\# \# \#}$ & $1.73 \pm 0.31^{\# \# \#}$ & $-1.73 \pm 0.34^{\# \# \#}$ \\
\hline Treated for 24 months & 38 & $29.59 \pm 4.97^{\# \# \Delta \Delta \Delta}$ & $14.58 \pm 3.82^{\# \# \# \Delta \Delta \Delta}$ & $1.99 \pm 0.36^{\# \# \Delta \Delta \Delta}$ & $-2.02 \pm 0.54^{\# \# \# \Delta \Delta}$ \\
\hline$P$ value & - & $<0.001^{\star \star \star}$ & $<0.001^{\star \star \star}$ & $<0.001^{\star \star \star}$ & $<0.001^{\star \star \star}$ \\
\hline
\end{tabular}

Comparison among before treatment, treated for 12 months and treated for 24 months, ${ }^{* * *} \mathrm{P}<0.001$. Compared with before treatment, ${ }_{\# \# \# P} \mathrm{P}<0.001$. Compared with treated for 12 months, ${ }^{\Delta \Delta} \mathrm{P}<0.01,{ }^{\Delta \Delta} \mathrm{P}<0.001 .2 \mathrm{D}-\mathrm{STI}$, two-dimensional speckle tracking imaging; $\mathrm{PAF}$, paroxysmal atrial fibrillation.

Table 7 Data change of RT-3DE examination of the patients in PAF observation group

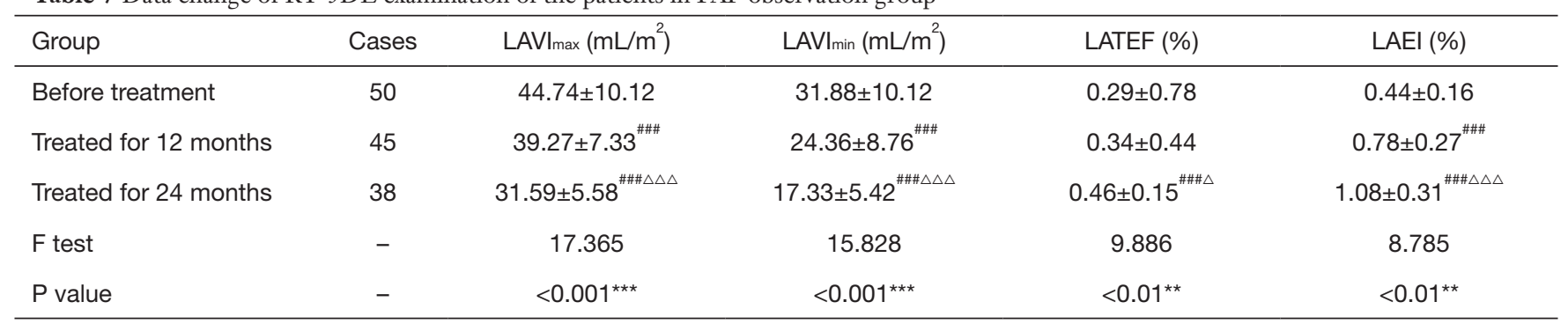

Comparison among before treatment, treated for 12 months and treated for 24 months, ${ }^{* *} \mathrm{P}<0.01$, ${ }^{* *} \mathrm{P}<0.001$. Compared with before treatment, ${ }^{\# \#} \mathrm{P}<0.001$. Compared with treated for 12 months, ${ }^{\Delta} \mathrm{P}<0.05,{ }^{\Delta \Delta \Delta} \mathrm{P}<0.001$. RT-3DE, real-time 3-dimensional echocardiography; PAF, paroxysmal atrial fibrillation.

ventricle). LAAEF, $\mathrm{mSa}$, and $\mathrm{mSRa}$ were used to evaluate this function.

Our results showed that the 'storage' and 'transport' functions of the left atrium of patients with ETH without PAF were reduced at the initial assessment. The 'storage' and 'transport' functions of the left atrium of patients with ETH combined with PAF were also reduced, and the auxiliary pump function disappeared. This may be because the ETH patients in this study were all old and the level of angiotensin-converting enzyme in the atrial tissue was significantly increased to trigger atrial remodeling. The increasing residual blood volume in the left atrium caused 
Table 8 Blood pressure and atrial fibrillation rate of the patients in PAF observation group during follow-up

\begin{tabular}{|c|c|c|c|c|c|}
\hline Time & Cases & \multicolumn{2}{|c|}{ Blood pressure (mmHg) } & \multicolumn{2}{|c|}{ Atrial fibrillation } \\
\hline Before treatment & 50 & $133.68 \pm 12.37$ & $80.55 \pm 10.38$ & - & - \\
\hline Treated for 12 months & 45 & $120.54 \pm 10.35^{\# \# \#}$ & $76.46 \pm 9.82$ & 8 & 16.33 \\
\hline Treated for 24 months & 38 & $114.33 \pm 9.54^{\# \# \Delta \Delta}$ & $74.25 \pm 9.52^{\# \#}$ & 13 & 34.21 \\
\hline$P$ value & - & $0.009^{\star *}$ & $0.005^{\star \star}$ & & - \\
\hline
\end{tabular}

Comparison among before treatment, treated for 12 months and treated for 24 months, ${ }^{\star \star} \mathrm{P}<0.01$. Compared with before treatment,

${ }^{\#} \mathrm{P}<0.01,{ }^{\# \#} \mathrm{P}<0.001$. Compared with treated for 12 months, ${ }^{\Delta \Delta} \mathrm{P}<0.01$. PAF, paroxysmal atrial fibrillation.

it to relax and expand, leading to a decrease in the function of 'storage' (29). At the same time, the left atrial passive emptying volume of the left atrium is reduced, causing 'transport' dysfunction. When PAF occurs in patients with ETH, the atrial muscle loses its normal contractile function due to uncoordinated atrial electrophysiological activities, which leads to the disappearance of left atrial auxiliary pump function and reduction of left atrial blood output, further leading to an increase in left atrial volume load and reduction in its storage function $(29,30)$. Changes to these three functions affect each other and further cause left atrial dysfunction in patients with ETH combined with PAF.

All patients with ETH combined with PAF in this study received telmisartan combined with rosuvastatin calcium and 2D-STI and RT-3DE technology were used to evaluate their therapeutic effect. During the 2-year follow-up, we found that atrial remodeling in patients with ETH combined with PAF had a significant reversal and cardiac function had been significantly restored. These patients still had atrial fibrillation after receiving the medication, but its frequency was low. Telmisartan is a new type of angiotensin II receptor blocker with a strong antihypertensive effect (31). It can effectively maintain balance of the renin-angiotensin-aldosterone system and inhibit the production of inflammatory mediators. Furthermore, it has a certain inhibitory effect on atrial remodeling and electrical remodeling. Angiotensin II receptor blockers play an important role in clinical blood pressure lowering treatment and telmisartan can also inhibit endogenous angiotensin. In addition, telmisartan can effectively prevent recurring atrial fibrillation. Several reasons have been forwarded to account for this effect $(32,33)$. Firstly, telmisartan can selectively block angiotensin type I receptors, thereby reducing the phenomenon of atrial myocardial calcium overload, reducing the myocardial shortening caused by high-frequency electrical stimulation of atrial fibrillation, and reducing atrium myoelectric remodeling. Secondly, it has a certain inhibitory effect on the renin-angiotensin-aldosterone system, which can block the collagen proliferation and myocardial fibrosis caused by angiotensin type I receptors. A third possible mechanism pertains to the ability of telmisartan to activate angiotensin II receptors, thereby effectively exerting vasodilation, anti-proliferation, anti-fibrosis and regulating apoptosis which can protect the heart, reverse left atrium myocardial hypertrophy, and improve diastolic function and reduce atrial remodeling. Rosuvastatin calcium is a common statin medication which can competitively inhibit the endogenous cholesterol synthesis rate-limiting enzyme reductase, block the intracellular mevalonic acid metabolism pathway, reduce intracellular cholesterol synthesis, and regulate blood lipid levels effectively $(34,35)$. Statins can also effectively stabilize plaques, regulate vascular endothelial function, and inhibit inflammation, oxidation, and the proliferation and migration of vascular smooth muscle cells, thereby improving myocardial remodeling (36).

The limitations to this research are (I) there was no separate analysis software for the left atrium and (II) we did not set up a control group to explore the therapeutic effect of telmisartan combined with rosuvastatin calcium because of the small number of patients.

In summary, 2D-STI and RT-3DE technologies could accurately and comprehensively evaluate changes in atrial remodeling and cardiac function. In patients with ETH combined with PAF, the left atrial 'storage' and 'transport' functions were reduced, the 'Auxiliary pump' function 
disappeared, and the stiffness of the left atrium increased. Telmisartan combined with rosuvastatin calcium treatment could significantly reverse these changes.

\section{Acknowledgments}

Funding: None.

\section{Footnote}

Reporting Checklist: The authors have completed the STROBE reporting checklist. Available at http://dx.doi. org/10.21037/jtd-20-3577

Data Sharing Statement: Available at http://dx.doi. org/10.21037/jtd-20-3577

Conflicts of Interest: All authors have completed the ICMJE uniform disclosure form (available at http://dx.doi. org/10.21037/jtd-20-3577). The authors have no conflicts of interest to declare.

Ethical Statement: The authors are accountable for all aspects of the work in ensuring that questions related to the accuracy or integrity of any part of the work are appropriately investigated and resolved. All procedures performed in this study involving human participants were in accordance with the Declaration of Helsinki (as revised in 2013). The study was approved by the Clinical Ethics Committee of The Central Hospital of Wuhan (No. CHW201609). All participating patients had been informed of its content and signed informed consent.

Open Access Statement: This is an Open Access article distributed in accordance with the Creative Commons Attribution-NonCommercial-NoDerivs 4.0 International License (CC BY-NC-ND 4.0), which permits the noncommercial replication and distribution of the article with the strict proviso that no changes or edits are made and the original work is properly cited (including links to both the formal publication through the relevant DOI and the license). See: https://creativecommons.org/licenses/by-nc-nd/4.0/.

\section{References}

1. Polivoda SN, Cherepok AA. Changes in activity of transcription factors in the cells of vascular endothelium as a pathological mechanism of its dysfunction in essential hypertension. Ter Arkh 2005;77:59-62.

2. Watts G. Neil Poulter: upping the pressure on hypertension. Lancet 2016;388:2585.

3. Chen Y, Zhu L, Fang Z, et al. Soluble guanylate cyclase contribute genetic susceptibility to essential hypertension in the Han Chinese population. Ann Transl Med 2019;7:620.

4. Bao XJ, Mao SQ, Gu TL et al. Hypomethylation of the Interferon gamma Gene as a Potential Risk Factor for Essential Hypertension: A Case-Control Study. Tohoku J Exp Med 2018;244:283-90.

5. Badran HM, Eid MA, Michael A. Doppler-derived indexes and B-type natriuretic peptide in prediction of paroxysmal atrial fibrillation in essential hypertension: a prospective study. Echocardiography 2007;24:911-22.

6. Prinsen JK, Kannankeril PJ, Sidorova TN et al. Highly

Reactive Isolevuglandins Promote Atrial Fibrillation

Caused by Hypertension. JACC Basic Transl Sci 2020;5:602-15.

7. Parcha V, Patel N, Kalra R et al. Incidence and Implications of Atrial Fibrillation/Flutter in Hypertension: Insights From the SPRINT Trial. Hypertension 2020;75:1483-90.

8. Suzuki K, Kato T, Koyama $S$ et al. Influence of Percutaneous Occlusion of Atrial Septal Defect on Left Atrial Function Evaluated Using 2D Speckle Tracking Echocardiography. Int Heart J 2020;61:83-88.

9. Yaman B, Akpinar O, Cerit L et al. Effects of chronic cigarette smoking on myocardial deformation parameters by two-dimensional speckle tracking echocardiography. Echocardiography 2019;36:2026-32.

10. Rakha S, Aboelenin HM. Left ventricular functions in pediatric patients with ten years or more type 1 diabetes mellitus: Conventional echocardiography, tissue Doppler, and two-dimensional speckle tracking study. Pediatr Diabetes 2019;20:946-54.

11. Kitano T, Nabeshima $Y$, Abe $Y$ et al. Accuracy and reliability of novel semi-automated two-dimensional layer specific speckle tracking software for quantifying left ventricular volumes and function. PLoS One 2019;14:e221204.

12. Zhang H, Qiu S, Chen F et al. Three-dimensional speckletracking echocardiography for evaluating myocardial motion in patients with cardiorenal syndrome. J Clin Ultrasound 2019;47:412-18.

13. Mohty D, Petitalot V, Magne J et al. Left atrial function in patients with light chain amyloidosis: A transthoracic 3D speckle tracking imaging study. J Cardiol 2018;71:419-27. 
14. Jularic M, Akbulak RO, Schaffer B et al. Image integration into 3-dimensional-electro-anatomical mapping system facilitates safe ablation of ventricular arrhythmias originating from the aortic root and its vicinity. Europace 2018;20:520-27.

15. Qin C, David Meggo-Quiroz L, Nanda NC et al. Early effect of essential hypertension on the left ventricular twistdisplacement loop by two-dimensional ultrasound speckle tracking imaging. Echocardiography 2014;31:631-7.

16. Wu J, Du X, Lv Q et al. A phase 3 double-blind randomized (CONSORT-compliant) study of azilsartan medoxomil compared to valsartan in Chinese patients with essential hypertension. Medicine (Baltimore) 2020;99:e21465.

17. Park SJ, Rhee SJ. Real-World Effectiveness and Safety of a Single-Pill Combination of Olmesartan/Amlodipine/ Hydrochlorothiazide in Korean Patients with Essential Hypertension (RESOLVE): A Large, Observational, Retrospective, Cohort Study. Adv Ther 2020;37:3500-14.

18. Naruse M, Koike Y, Kamei N et al. Effects of azilsartan compared with telmisartan on insulin resistance in patients with essential hypertension and type 2 diabetes mellitus: An open-label, randomized clinical trial. PLoS One 2019;14:e0214727.

19. Zhao D, Liu H, Dong P. A Meta-analysis of antihypertensive effect of telmisartan versus candesartan in patients with essential hypertension. Clin Exp Hypertens 2019; 41: 75-79.

20. Yan Y, Li XL. Evaluation of Left Atrial Structure and Function with Two-dimensional Speckle Tracking Imaging and Real-time Three-dimensional Imaging in Patients with Paroxysmal Atrial Fibrillation After Radiofrequency Catheter Ablation. Sichuan Da Xue Xue Bao Yi Xue Ban 2019;50:390-95.

21. Roeh A, Schuster T, Jung $P$ et al. Two dimensional and real-time three dimensional ultrasound measurements of left ventricular diastolic function after marathon running: results from a substudy of the BeMaGIC trial. Int J Cardiovasc Imaging 2019;35:1861-69.

22. Georgiopoulos G, Tsioufis C, Kalos T et al. Serum Uric Acid is Independently Associated with Diastolic Dysfunction in Apparently Healthy Subjects with Essential Hypertension. Curr Vasc Pharmacol 2019;17:99-106.

23. La Salvia S, Gunasekaran PM, Byrd JB et al. Extracellular Vesicles in Essential Hypertension: Hidden Messengers. Curr Hypertens Rep 2020;22:76.

24. Xu L, Wang N, Chen X et al. Quantitative evaluation of myocardial layer-specific strain using two-dimensional speckle tracking echocardiography among young adults with essential hypertension in China. Medicine (Baltimore) 2018;97:e12448.

25. Xu M, Liu F, Ge ZX et al. Functional studies of left atrium and BNP in patients with paroxysmal atrial fibrillation and the prediction of recurrence after CPVA. Eur Rev Med Pharmacol Sci 2020;24:4997-5007.

26. Atas H, Kepez A, Tigen K et al. Evaluation of left atrial volume and function in systemic sclerosis patients using speckle tracking and real-time three-dimensional echocardiography. Anatol J Cardiol 2016;16:316-22.

27. Zhao Z, Wang X, Wang S et al. Research based on the core pathogenesis in the treatment according to traditional Chinese medicine syndrome differentiation for heart failure with normal ejection fraction. Medicine (Baltimore) 2020;99:e21663.

28. Shah S, Henry A, Roselli C et al. Genome-wide association and Mendelian randomisation analysis provide insights into the pathogenesis of heart failure. Nat Commun 2020;11:163.

29. Polovina MM, Coats A, Seferovic P. Is left atrium the best kept secret of the heart? Left atrial dilatation and cardiovascular outcomes. Heart 2019;105:1848-49.

30. Kishima H, Mine T, Fukuhara E et al. Is the abnormal conduction zone of the left atrium a precursor to a low voltage area in patients with atrial fibrillation? J Cardiovasc Electrophysiol 2020;31:2874-82.

31. Yamashita T, Ogawa S, Aizawa Y et al. Randomized study of angiotensin II type 1 receptor blocker vs dihydropiridine calcium antagonist for the treatment of paroxysmal atrial fibrillation in patients with hypertension. Circ J 2006;70:1318-21.

32. Li J, Chen H, Zhou Q et al. Effects of telmisartan on paroxysmal atrial fibrillation in hypertensive patients. Pak J Pharm Sci 2017;30:1651-55.

33. Wang WW, Zhang FL, Chen JH et al. Telmisartan reduces atrial arrhythmia susceptibility through the regulation of RAS-ERK and PI3K-Akt-eNOS pathways in spontaneously hypertensive rats. Can J Physiol Pharmacol 2015;93:657-65.

34. Lv Q, Wang Y, Li Y et al. Rosuvastatin Reverses Hypertension-Induced Changes in the Aorta Structure and Endothelium-Dependent Relaxation in Rats Through Suppression of Apoptosis and Inflammation. J Cardiovasc Pharmacol 2020;75:584-95.

35. Kim TS, Rha SW, Kim SY et al. Efficacy and 
Tolerability of Telmisartan/Amlodipine and Rosuvastatin Coadministration in Hypertensive Patients with Hyperlipidemia: A Phase III, Multicenter, Randomized, Double-blind Study. Clin Ther 2019;41:728-41.

36. Park JS, Jeon JY, Kim MG. Pharmacokinetic and bioequivalence study of a fixed-dose combination of

Cite this article as: Jiang $\mathrm{F}$, Chen $\mathrm{Y}, \mathrm{Wu} \mathrm{L}$, Zhang Y, Liu J, Sun X, Li J, Mao M, Yang S. Left heart function evaluation of patients with essential hypertension and paroxysmal atrial fibrillation by two-dimensional speckle tracking imaging combined with real-time three-dimensional ultrasound. J Thorac Dis 2021;13(1):322-333. doi: 10.21037/jtd-20-3577 amlodipine besylate and rosuvastatin calcium compared to co-administration of separate tablets in healthy Korean subjects. Int J Clin Pharmacol Ther 2019;57:612-22.

(English Language Editor: B. Draper) 